\title{
A randomised crossover comparison of two endotracheal tube introducers: the FROVA and the Flexible Tip Bougie for GlideScope intubation of a difficult airway manikin by infrequent intubators
}

\author{
John Cormack ${ }^{1,2^{*}}$ (D) Bridget Langley ${ }^{1,2}$, Louisa-Rose Bhanabhai ${ }^{2}$ and Roman Kluger $^{1,2}$
}

\begin{abstract}
Background: This unblinded randomised crossover study compares two endotracheal tube introducers (ETIs): the FROVA and the "Flexible Tip Bougie" (FTB), in an airway manikin mimicking difficult intubation with a percentage of glottic opening view of 30\%. Participants were Emergency Medicine and Anaesthesia trainees with recent experience of less than twenty patient intubations. The primary outcome was time to intubation, further divided into time taken to pass the ETI and time to railroad the endotracheal tube (ETT) over the ETI. The secondary outcome was the difficulty of intubation.

Results: The median total time to ETT placement was significantly shorter with the FTB (37.5 s) compared with the FROVA ETI $(63.0 \mathrm{~s}), P=0.0006$. The median difficulty reported (scores $0-10$ with 0 being no difficulty) with the FTB was 2 compared with 5 for the FROVA, $P<0.0001$.

Conclusions: The FTB enabled significantly faster and easier placement of the endotracheal tube compared with the FROVA in inexperienced hands intubating a difficult intubation manikin.
\end{abstract}

Keywords: Intratracheal intubation, Manikin, Teaching materials, Airway management

\section{Introduction}

\section{Background}

Intubation is challenging for the novice intubator in the emergency department where suboptimal positioning, urgency and injuries can add to the difficulty. The GlideScope (Verathon, Bothell, WA) has given novices an improved tool compared with traditional Macintosh blade direct laryngoscopy [1-3], allowing a rapid learning curve, less cervical spine movement and fewer oesophageal

\footnotetext{
* Correspondence: cormackj@ozemail.com.au

${ }^{1}$ The Faculty of Medicine, Dentistry and Health Sciences, University of Melbourne, Building 181, Grattan St, Melbourne 3010, Australia 2Department of Anaesthesia and Acute Pain Medicine, St Vincent's Hospital Melbourne, 41 Victoria Parade, PO Box 2900, Fitzroy VIC 3065, Australia
}

intubations [4]. The problem of anterior tracheal impingement, causing the "can see but can't intubate" phenomenon, is a common issue and while most intubations are eventually achieved; this may involve multiple attempts with the possibility of laryngeal trauma or hypoxia.

Devices to assist GlideScope intubation include stylets and endotracheal tube introducers (ETIs) (bougies), and the latter have been used to improve intubation success when difficulty is encountered [5]. These static guides can be bent into a curve which cannot be altered during each intubation attempt. Alternatively, some ETIs have a flexible tip replicating the action of a flexible bronchoscope (fiberscope) tip. When a fiberscope is used by

(c) The Author(s). 2020 Open Access This article is licensed under a Creative Commons Attribution 4.0 International License, which permits use, sharing, adaptation, distribution and reproduction in any medium or format, as long as you give appropriate credit to the original author(s) and the source, provide a link to the Creative Commons licence, and indicate if changes were made. The images or other third party material in this article are included in the article's Creative Commons licence, unless indicated otherwise in a credit line to the material. If material is not included in the article's Creative Commons licence and your intended use is not permitted by statutory regulation or exceeds the permitted use, you will need to obtain permission directly from the copyright holder. To view a copy of this licence, visit http://creativecommons.org/licenses/by/4.0/ The Creative Commons Public Domain Dedication waiver (http://creativecommons.org/publicdomain/zero/1.0/) applies to the data made available in this article, unless otherwise stated in a credit line to the data. 
experienced intubators as a "dynamic introducer", it is superior to a stylet in difficult GlideScope intubation [6].

A commonly used ETI is the FROVA (Cook Medical, Bloomington, IN) which can be bent into a curve matching that of the GlideScope and placed in the trachea. Once the ETI is intratracheal, it is used as a guide for the endotracheal tube (ETT) to pass over (railroad). It is hollow allowing oxygenation if needed. When space is limited or the glottic view is reduced, an ETI allows a clearer view of the tip entering the larynx due to its small diameter. The "Flexible Tip Bougie" (FTB, Construct Medical, Hawthorn, $\mathrm{VIC}$ ) has a permanent curve matching the GlideScope and the ability to flex or retroflex at the tip using pulsion or traction on a low-profile slide on the shaft (Fig. 1). A previous difficult airway manikin study has compared these two devices with experienced intubators and found that the FTB was superior with reduced time to intubation and easier intubation [7].

\section{Importance}

The global corona virus disease 19 (COVID-19) pandemic has created an unprecedented demand for rapid and successful endotracheal intubation, often under unfavourable conditions of hypoxia in locations including intensive care and the emergency department. The intubation may need to be performed by staff who have previously only intubated occasionally, with ideal conditions and under supervision. The study was devised prior

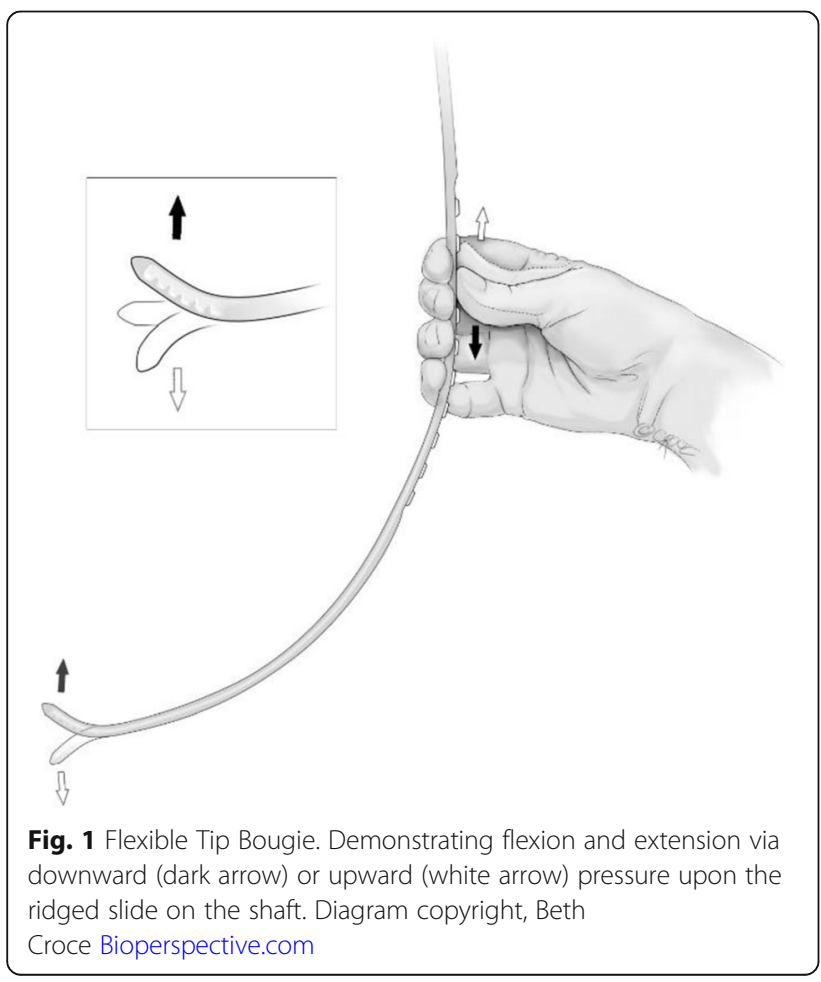

to this global emergency; however, the aim to help define the fastest and easiest intubation method for the novice intubator under difficult circumstances is more relevant than ever.

\section{Goals}

Our hypothesis was that Glidescope intubation aided by the FTB would be quicker and easier to use than the FROVA with inexperienced intubators.

\section{Methods}

\section{Study design and setting}

A single centre randomised, unblinded crossover trial approved by the institutional Ethics Board (LRR171/18).

\section{Selection of participants}

Thirty novice intubators were recruited, including Emergency Medicine trainees and Anaesthetic residents. All trainees able to attend during normal working hours were invited to participate. The exclusion criterium was greater than twenty patient intubations in the previous calendar year. Five enrolled participants were unable to attend during the specified times. One participant revealed greater experience and was excluded from the analysis. Twenty-four participants completed the study and were included for analysis. A flow chart from enrolment to analysis is shown (Fig. 2).

\section{Interventions}

Participants were consented, given instructions and shown an instructional video demonstrating both devices. The video was reviewed on the study day, and questions were invited. Each participant was given the opportunity to examine both ETIs before commencing.

The AirSim Advance X manikin (Trucorp, Lurgan, N. Ireland) was positioned in the simulation centre or in a quiet room within the operating theatre complex. The GlideScope/manikin setup offered a percentage of glottic opening (POGO) view of 30\% [8], unchanged during the study period. Silicone lubricant was applied to each ETI. The GlideScope was a Cobolt AVL model with a size 4 GVL Stat hyperangulated blade (Verathon, BC, Canada) fixed into position throughout the study on a frame attached to the base of the manikin (Fig. 3). Subsequent MacIntosh laryngoscopy revealed a Cormack and Lehane view grade three [9].

Participants were randomised to either the FROVA or FTB first. Total time to intubation was the time from receiving the ETI until the ETT was placed in the midtrachea and was further broken down into two components: the time to placement of the ETI and time from placement of ETI until placement of the ETT (Appendix: supplementary video 1). Difficulty was rated for each phase on a scale from zero to ten (ten $=$ most difficult). 


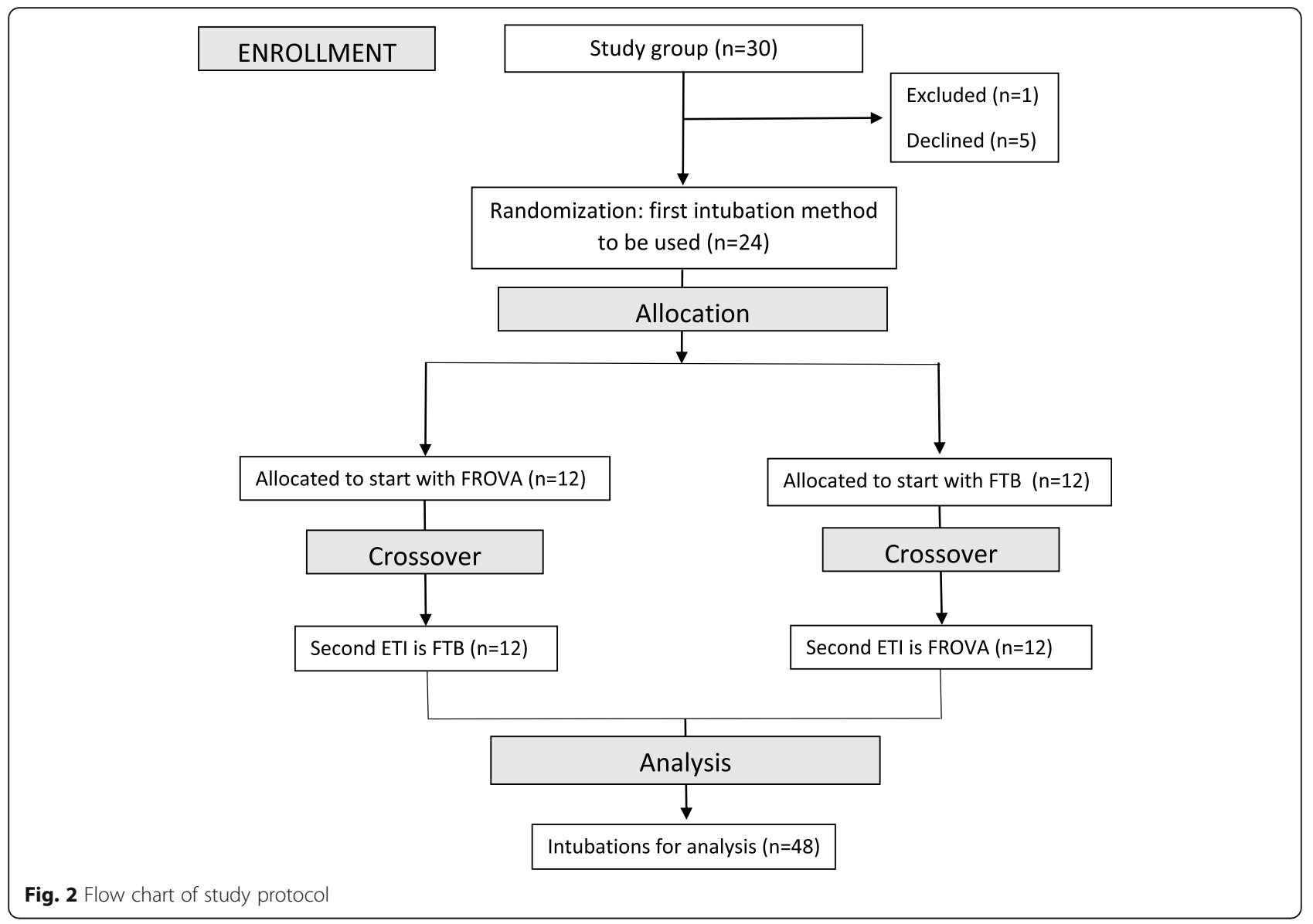

Five minutes was the arbitrary point at which intubation attempts would be abandoned. Interviews were conducted at completion.

The second phase of the crossover study was a replication of the first phase using the remaining ETI type (either FROVA or FTB). The order of ETI used was randomised in permuted blocks of four. The numbers for the study were derived from a similar study looking at time to intubation comparing the same two ETIs with experienced intubators ${ }^{7}$.

\section{Measurement}

With the backup of audiovisual recording, an investigator stood beside the participant, passing the equipment and was responsible for determining and signalling the insertion of the ETI and then the ETT by watching the GlideScope screen. A second investigator was observing and recording times for each endpoint. A third investigator reviewed video recordings to confirm accuracy of endpoint times.

\section{Outcomes}

Time until the ETT reached the mid-trachea was the primary outcome, broken down into two component parts: the time to insert the ETI into the mid trachea and the time to fully insert the ETT over the ETI (Supplementary video 1). Secondary outcomes were difficulty of ETI insertion and difficulty of ETT passage over the ETI. We also collected descriptive data to highlight any specific problems encountered.

Upon review of the descriptive data and audiovisual recordings, the most frequent difficulty with the FROVA was that the tip impinged upon the anterior tracheal wall $(5 / 24)$ preventing further insertion to the mid trachea and making it impossible to advance the ETT beyond the impingement. This did not occur with the FTB $(0 / 24)$ as the impingement was prevented by extending the tip down towards the trachea. In all cases, the time to placement of the ETI was recorded as the time to reach mid-trachea regardless of whether one or more attempts at ETT placement were made prior to this being achieved.

We measured the performance of the ETI device and eliminated the skill of visualising the larynx. Fixing the GlideScope in a static position throughout ensured a consistent view of the glottis during the study and removed a laryngoscopy learning effect favouring the second arm. Dividing the intubation time into two epochs was important to discern if the process of railroading the tube over the ETI was different between the two devices. 


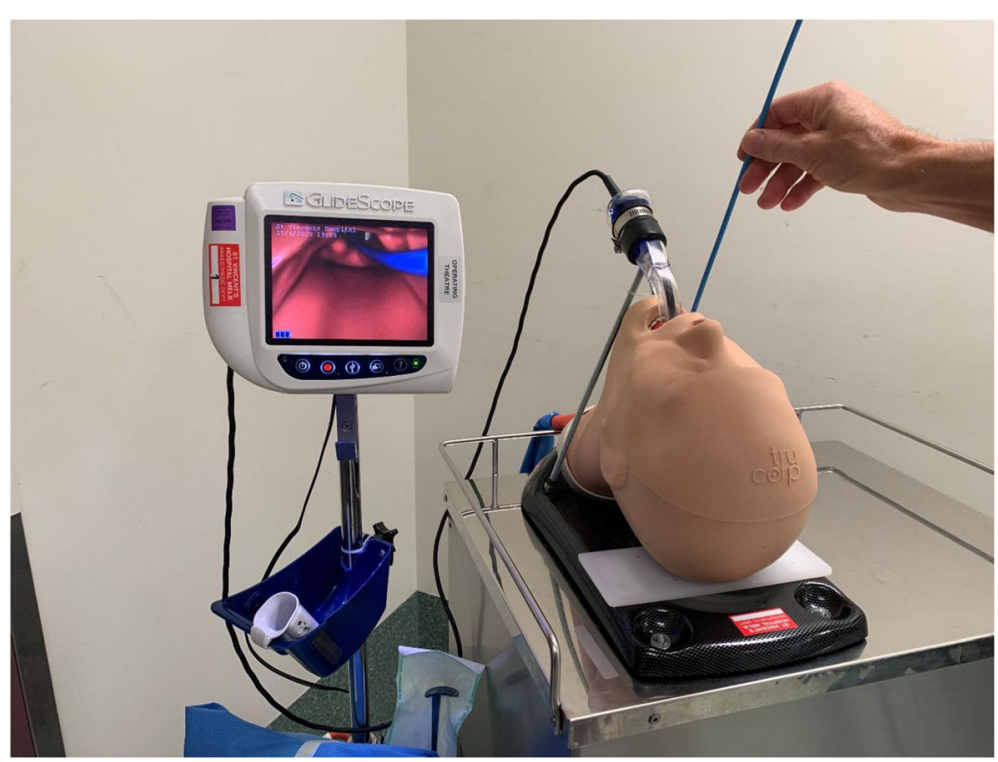

Fig. 3 AirSim Advance X Manikin modified to extend the head and with tongue insert fully inflated. GlideScope held in static position by rods attached to the base of the manikin

Paper data entry was transcribed by a research nurse onto an Excel database (Microsoft) and rechecked for accuracy against the paper record by the principle investigator. Spurious results were compared with the video and digital clock recordings.

\section{Analysis}

Times and difficulty scores were compared between groups using Wilcoxon matched pairs tests as the data was not normally distributed. A $P$ value $<0.05$ was deemed significant. All statistical analyses were performed using Stata/IC 14.2 for Windows, StataCorp LLC, TX, USA.

\section{Results}

\section{Characteristics of study subjects}

Emergency Medicine trainees were in year one to four and Anaesthetic residents were in their second year of residency, rotating through an anaesthesia term. Many had not recorded human and manikin intubation numbers; however, strict assurance of less than 20 human intubations in the previous 12 months was required in all cases. Characteristics of the participants experience are summarised (Table 1).

\section{Main results}

The median total time to endotracheal tube placement was significantly shorter with the FTB (37.5 s) compared with the FROVA ETI (63.0 s), $P=0.0006$. One participant using the FROVA and one using the FTB were stopped at $5 \mathrm{~min}$ without being able to place the ETT in the mid trachea. These were both recorded as $300 \mathrm{~s}$ and were included in the analyses. The advantage of the FTB was due to quicker placement of the ETI rather than a difference in time to railroad the ETT. Median time to place ETI with FTB was $15 \mathrm{~s}$ and with FROVA $36.5 \mathrm{~s}(P=0.0001)$. Median time from placement of ETI until ETT was within the trachea with the FTB was $20 \mathrm{~s}$ and with the FROVA $16 \mathrm{~s}(P=0.9)$ (Fig. 4$)$.

The median difficulty $(0-10)$ of placing the ETI was significantly in favour of the FTB $=2$ compared with FROVA $=5(P=<0.001)$. For the passage of the endotracheal tube over the ETI, the median difficulty with the FTB was 3 compared with FROVA $4(P=$ 0.02) (Fig. 5).

The median difference in total time to intubation between the two ETIs was $25.5 \mathrm{~s}$; however, this varied with which ETI was used first, $44.5 \mathrm{~s}$ (FROVA first) vs $15.5 \mathrm{~s}$ (FTB first), probably indicating a learning effect (Table 2).

Table 1 Participant demographic data

\begin{tabular}{ll}
\hline Mean age (years) & 29.7 \\
Mean experience (months) & 22.3 \\
Mean solo intubations & 4.8 \\
Trainee stream & \\
Anaesthetic residents & $n=7$ \\
Emergency registrars & $n=17$ \\
Previous FROVA use & $22 / 24$ \\
Previous FTB use & $1 / 24$ \\
\hline
\end{tabular}




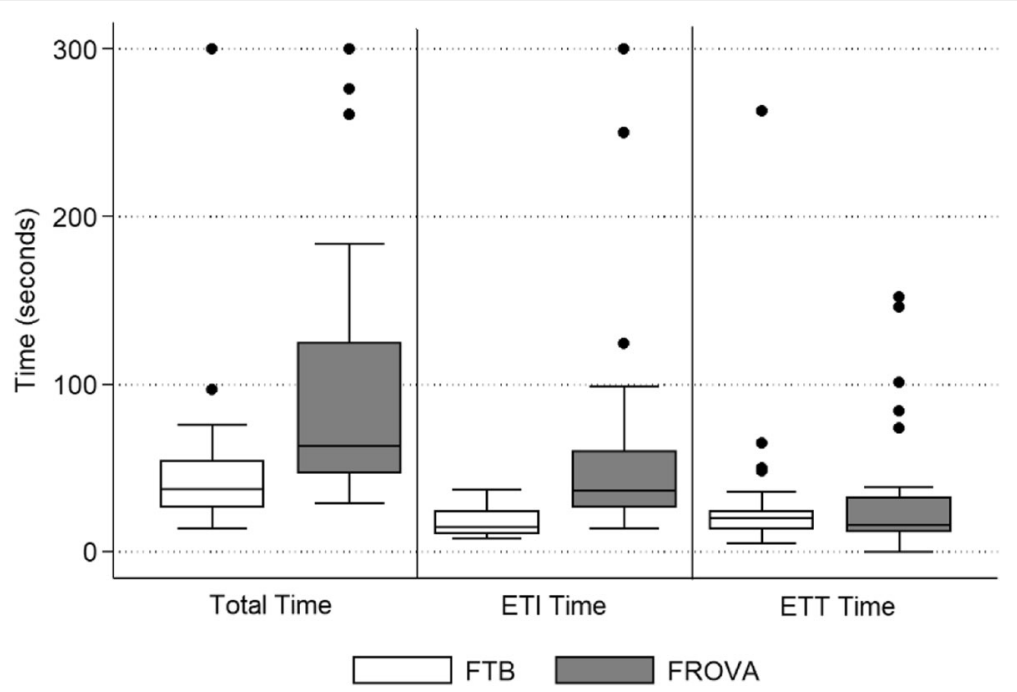

Fig. 4 Left graph: total time from receiving the ETI until ETT fully inserted. Central graph: time until ETI reached mid trachea. Right graph: time to railroad ETT over the ETI

\section{Discussion}

An advantage of the FTB compared with the FROVA both in time taken (median 37.5 vs $63 \mathrm{~s}$ ) and ease of intubation was demonstrated. This is due to the ETI placement (median 15 vs $36.5 \mathrm{~s}$ ), not subsequent railroading of the ETT. This advantage was reduced but still significant in the group using the FTB first. Familiarity with the FROVA device (previous FROVA use by $22 / 24$ participants) may have been expected to contribute to improved performance with this device and may have biased the study from finding an even greater advantage with the FTB.

In a retrospective data analysis of first pass and overall intubation success, Mosier et al. studied difficulty associated with emergency department intubations revealing an
82\% first pass success rate with the GlideScope [10]. Sakles et al. reported a close association between adverse events and multiple attempts at intubation in the emergency department [11]. With experience, ETIs (bougies) have been useful in MacIntosh laryngoscopy and intubation, but with the GlideScope, the bougie has not made a significant improvement $[12,13]$, compared with a stylet.

The GlideScope's decreased working space and acute laryngeal entry angle compared with the MacIntosh laryngoscope has led to investigation of the "can see but can't intubate" situation. Case reports have suggested that the flexible tip of a videobronchoscope (fiberscope) can prevent ETT impingement on the trachea $[14,15]$, and a prospective clinical trial

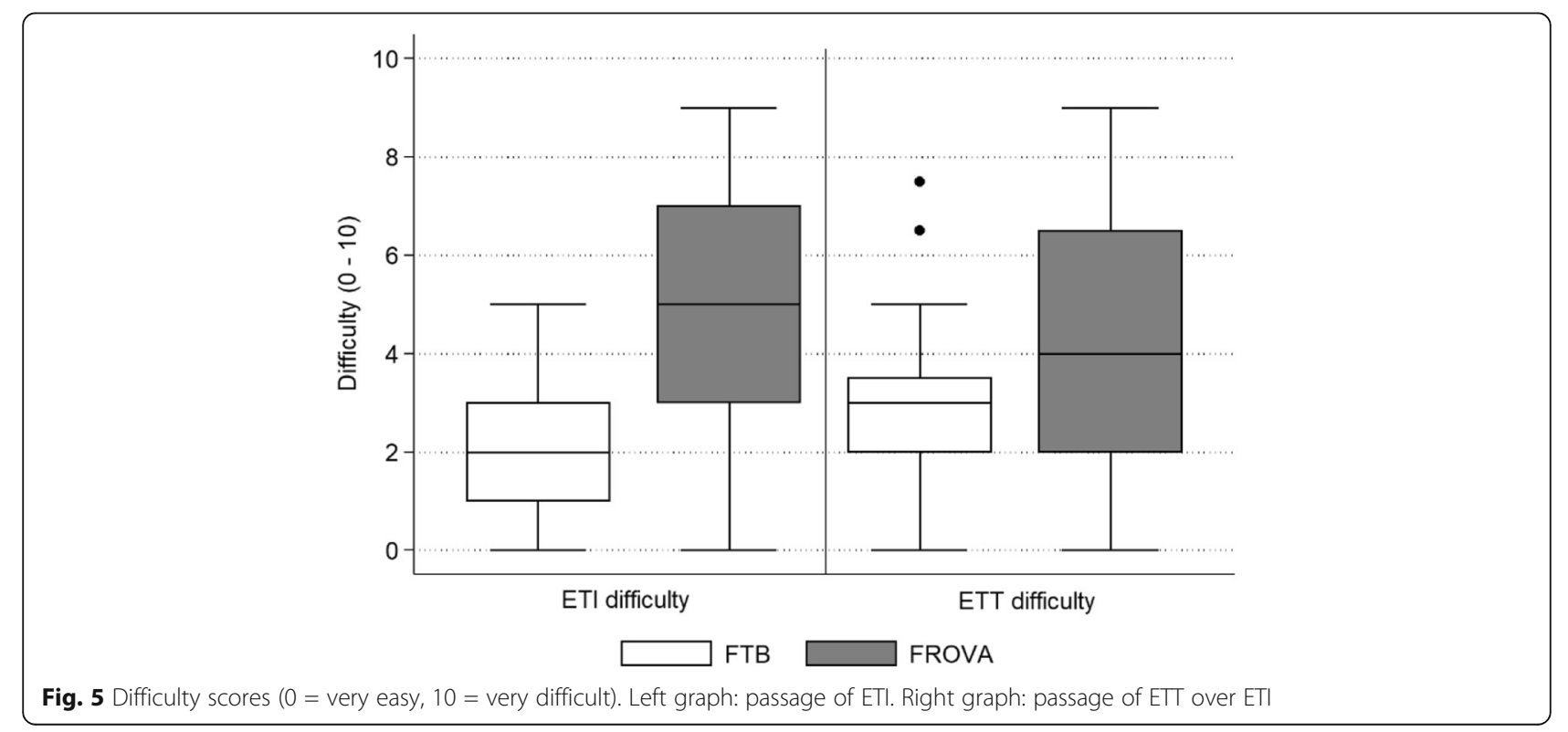


Table 2 A learning effect favouring the second ETI used

\begin{tabular}{|c|c|c|c|c|c|}
\hline \multicolumn{6}{|c|}{ Median times in seconds } \\
\hline \multicolumn{6}{|c|}{ All participants $(n=24)$} \\
\hline ETI type & ETI time** & ETT time & Total time** & ETI difficulty** & ETT difficulty* \\
\hline FTB & 15 & 20 & 37.5 & 2 & 3 \\
\hline FROVA & 36.5 & 16 & 63 & 5 & 4 \\
\hline \multicolumn{6}{|c|}{ FROVA ETI first group $(n=12)$} \\
\hline ETI type & ETI time** & ETT time & Total time** & ETI difficulty* & ETT difficulty* \\
\hline FTB & 11 & 18.5 & 29.5 & 2 & 2.5 \\
\hline FROVA & 41 & 16 & 74 & 5 & 5 \\
\hline \multicolumn{6}{|c|}{ FTB ETI first group $(n=12)$} \\
\hline ETI type & ETI time** & ETT time & Total time & ETI difficulty* & ETT difficulty \\
\hline FTB & 20 & 20.5 & 38.5 & 2 & 3 \\
\hline FROVA & 36.5 & 14.5 & 54 & 5 & 3.5 \\
\hline
\end{tabular}

${ }^{*} P<0.01 ; * P<0.05$

showed that a flexible tip ETI (fiberscope) was superior to a preshaped stylet [6]. A comparison of the FROVA and the FTB with experienced intubators using the GlideScope showed faster and easier intubation with the FTB [7], and another manikin study using a MacIntosh laryngoscope showed superiority of the FTB during simulated cardiac arrest [16]. The FTB provides a cheaper, accessible alternative to the fiberscope, requiring only one intubator. Despite its recent introduction and a paucity of studies, the FTB is already listed on an emergency department COVID-19 intubation trolley [17].

\section{Limitations}

Manikin studies can only approximate clinical conditions, but they are justifiable and ethical when a new device is used with novice intubators [18]. The $30 \%$ glottic view and an anterior larynx (seen at the upper border of a GildeScope screen) is reproducible with various models of manikin and adaptations to increase difficulty, as long as the laryngoscope is fixed. We designed the study so that the laryngoscopic view, the acute angle to be negotiated into the trachea and the friction encountered were reproducible and realistic.

Our "time to intubation" excludes laryngoscopy time. Sharma outlined the limitations of GlideScope "intubation" as opposed to "laryngoscopy", suggesting that the two should be considered separately [19]. Previous comparative manikin studies include laryngoscopy time, measuring the time the blade enters the mouth until the ETT is fully inserted $[12,16,20]$. By excluding laryngoscopy and providing a static, constant view of the larynx, we provided the same view for each participant in both arms of the crossover study. Therefore, the time to ETI insertion is neither likely to be primarily due to the variable laryngoscopy skill of the intubator, nor a laryngoscopy learning effect with the second device used. The laryngoscopy variability in novices may have been greater than the difference found between the ETIs studied, decreasing the power.

The study was unblinded. Blinding would have increased complexity possibly introducing errors of recording or processing.

\section{Conclusions}

In summary, our study examines a specific (yet common) difficult intubation problem which requires an initial sharp angulation anteriorally of the ETI or ETT into the larynx and then a redirection downwards into the trachea in order to achieve intubation. In this simulated situation, the FTB is superior to the FROVA. COVID-19 has created a global need for efficient intubation under difficult circumstances by intubators of variable experience. Historically, comparative quality analysis and then prospective clinical trials have followed case reports and manikin studies when a new device has shown an advantage worth pursuing.

\section{Supplementary information}

Supplementary information accompanies this paper at https://doi.org/10. 1186/s12245-020-00298-6.

Additional file 1: Supplemental video 1.

Abbreviations

ETI: Endotracheal tube introducer = "Bougie"; ETT: Endotracheal tube; FTB: Flexible Tip Bougie; COVID-19: Corona virus disease 19 


\section{Acknowledgements}

Dr. Caitlan Brabazon, Ms Petrea Corcoran and Ms Emily Fitzgerald for work with enrollment, data collection and performance of study protocols. Dr. Ian Summers for help with study design.

\section{Authors' contributions}

$J C, B L$ and $L B$ contributed to the concept development, equipment procurement, study design, protocol performance and editorial review. JC was the primary author with developmental concepts and outcome prioritisation by RK. Statistical review, data collation and statistical analyses by RK. All authors have read and approved the final manuscript.

\section{Funding}

There was no grant associated with the study. Equipment and use of the hospital SIM centre and research nurse time was provided by the Department of Anaesthesia.

\section{Availability of data and materials}

The data is available in paper and electronic database form and is securely stored in locked and password protected form in our hospital department (Department of Anaesthesia and Acute Pain Medicine, St. Vincent's Hospital Melbourne). The datasets generated during and/or analysed during the current study are available from the corresponding author on reasonable request.

\section{Ethics approval and consent to participate}

Institutional Ethics Board approval (LRR171/18) and written consent from each participant were obtained. Consent for publication was granted by all participants.

\section{Competing interests}

The authors declare that they have no competing interests.

Received: 14 May 2020 Accepted: 23 June 2020

Published online: 17 July 2020

\section{References}

1. Sakles JC, Kalin L. The effect of stylet choice on the success rate of intubation using the GlideScope video laryngoscope in the emergency department. Acad Emerg Med. 2012;19(2):235-8.

2. Kim HJ, Chung SP, Park IC, Cho J, Lee HS, Park YS. Comparison of the GlideScope video laryngoscope and Macintosh laryngoscope in simulated tracheal intubation scenarios. Emerg Med J. 2008;25(5):279-82.

3. Nouruzi-Sedeh P, Schumann M, Groeben H. Laryngoscopy via Macintosh blade versus GlideScope: success rate and time for endotracheal intubation in untrained medical personnel. Anesthesiology. 2009;110(1):32-7.

4. Sakles JC, Javedani PP, Chase E, Garst-Orozco J, Guillen-Rodriguez JM, Stolz $U$. The use of a video laryngoscope by emergency medicine residents is associated with a reduction in esophageal intubations in the emergency department. Acad Emerg Med. 2015;22(6):700-7.

5. Ciccozzi A, Angeletti C, Guetti C, Papola R, Angeletti PM, Paladini A, et al. GlideScope and Frova introducer for difficult airway management. Case Rep Anesthesiol. 2013;2013:717928.

6. Mazzinari G, Rovira L, Henao L, Ortega J, Casasempere A, Fernandez Y, et al. Effect of dynamic versus stylet-guided intubation on first-attempt success in difficult airways undergoing Glidescope laryngoscopy: a randomized controlled trial. Anesth Analg. 2019.

7. Brunckhorst TL, Grimmett W, Mun-San Kwan MM. Evaluation of a new bougie design for the difficult airway: a manikin crossover trial. Anaesth Intensive Care. 2018;46(5):544-5.

8. Levitan RM, Ochroch EA, Kush S, Shofer FS, Hollander JE. Assessment of airway visualization: validation of the percentage of glottic opening (POGO) scale. Acad Emerg Med. 1998;5(9):919-23.

9. Cormack RS, Lehane J. Difficult tracheal intubation in obstetrics. Anaesthesia 1984;39(11):1105-11.

10. Mosier J, Chiu S, Patanwala AE, Sakles JC. A comparison of the GlideScope video laryngoscope to the C-MAC video laryngoscope for intubation in the emergency department. Ann Emerg Med. 2013;61(4):414-20 e1.

11. Sakles JC, Chiu S, Mosier J, Walker C, Stolz U. The importance of first pass success when performing orotracheal intubation in the emergency department. Acad Emerg Med. 2013;20(1):71-8.
12. Nielsen AA, Hope CB, Bair AE. GlideScope videolaryngoscopy in the simulated difficult airway: bougie vs standard stylet. West J Emerg Med. 2010;11(5):426-31.

13. Batuwitage B, McDonald A, Nishikawa K, Lythgoe D, Mercer S, Charters P. Comparison between bougies and stylets for simulated tracheal intubation with the C-MAC D-blade videolaryngoscope. Eur J Anaesthesiol. 2015;32(6): 400-5.

14. Loh PS, Ng KWS. Combining glidescope and fiber-optic for intubation in oral maxillofacial surgery. J Anaesthesiol Clin Pharmacol. 2017;33(2):254-5.

15. Cormack JR, Ho ML. Combined use of C-MAC(R) videolaryngoscope and nasal videobronchoscope for critical airway rescue and intubation. Anaesth Intensive Care. 2018;46(3):347-8.

16. Evrin T, Smereka J, Gorczyca D, Bialka S, Ladny JR, Katipoglu B, et al. Comparison of different intubation methods in difficult airways during simulated cardiopulmonary resuscitation with continuous chest compression: a randomized cross-over manikin trial. Emerg Med Int. 2019; 2019:7306204.

17. Weingart, S (EMCRIT). Covid intubation packs https:/emcrit.org/emcrit/ covid19-intubation-packs-and-preoxygenation-for-intubation/. Accessed May 2020

18. Ward PA, Irwin MG. Man vs. manikin revisited - the ethical boundaries of simulating difficult airways in patients. Anaesthesia. 2016:71(12):1399-403.

19. Sharma D. Is GlideScope the best way to intubate? Anesthesiology. 2010; 113(1):258-9 author reply 9.

20. Gregory P, Woollard M, Lighton D, Munro G, Jenkinson E, Newcombe RG, et al. Comparison of malleable stylet and reusable and disposable bougies by paramedics in a simulated difficult intubation. Anaesthesia. 2012;67(4): $371-6$.

\section{Publisher's Note}

Springer Nature remains neutral with regard to jurisdictional claims in published maps and institutional affiliations.

Ready to submit your research? Choose BMC and benefit from:

- fast, convenient online submission

- thorough peer review by experienced researchers in your field

- rapid publication on acceptance

- support for research data, including large and complex data types

- gold Open Access which fosters wider collaboration and increased citations

- maximum visibility for your research: over $100 \mathrm{M}$ website views per year

At BMC, research is always in progress.

Learn more biomedcentral.com/submissions 